\title{
Negative perceptions of parental smoking among 61810 Hong Kong adolescents: A cross sectional school based survey
}

\author{
Jion Jiu Chen ${ }^{I}$, Sai Yin Ho ${ }^{I}$, Man Ping Wong ${ }^{2}$, Tai Hing Lom
}

\begin{abstract}
INTRODUCTION The present study aimed to investigate the negative perceptions of paternal and maternal smoking and their associated factors among adolescents in Hong Kong. METHODS In a school-based cross-sectional survey in 2010 to 2011, 61,810 Hong Kong secondary school students (mean age 14.6 years, $50.8 \%$ boys) reported whether their parents smoked and whether they considered their parents' smoking unacceptable and minded if their peers knew that their father or mother smoke (indicators of the negative perceptions). Factors associated with negative perceptions of paternal and maternal smoking were explored using multivariable regression models.

RESULTS Among students that reported paternal smoking ( $\mathrm{n}=19.184,29.8 \%$ ), 50.8\% considered it unacceptable, and $21.0 \%$ minded if their peers knew of it. Of those reporting maternal smoking $(\mathrm{n}=3,678,5.7 \%), 48.1 \%$ considered it unacceptable, and $30.4 \%$ minded if their peers knew of it. Generally, the indicators of negative perceptions of parental smoking were associated with younger age, being certain about the harm of smoking and secondhand smoke, no peer smoking, and no secondhand smoke from the respective parent. Considering parental smoking unacceptable was additionally associated with lower family affluence and not living with any smokers apart from the respective parent.

conclusions About half of adolescents in Hong Kong with a smoking parent considered their parental smoking unacceptable, and about a quarter minded if their peers knew of their parent's smoking. Such negative perceptions were more common in adolescents who were certain about the harm of tobacco, and had fewer co-residing smokers and no smoking peers.
\end{abstract}

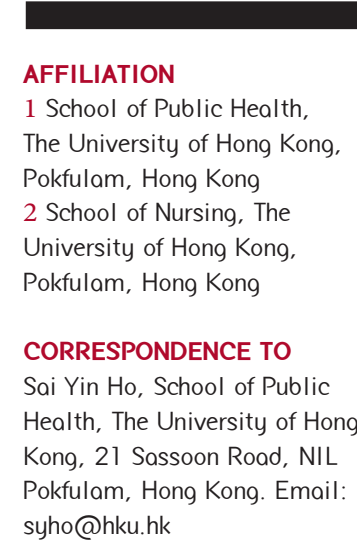

KEYWORDS

Perception, Parental smoking, Adolescents, Chinese

\section{INTRODUCTION}

Living with smokers may harm children. Secondhand smoke (SHS) has been causally linked to health consequences including lower respiratory tract illness, middle ear disease, more severe asthma and impaired lung function1. SHS and thirdhand smoke (THS) may also trigger physical discomfort in children, such as coughing and nausea ${ }^{2,3}$. Moreover, parental smoking predicts smoking in children, through imitation and easy access to cigarettes ${ }^{4,5}$. Tobacco kills up to two-thirds of smokers who started young. However, how children and adolescents themselves think about parental smoking is relatively understudied.

To our knowledge, children or adolescents' perceptions of family members' smoking status were reported in a few studies, all of which were qualitative and conducted in the West ${ }^{7-9}$. A study in the United Kingdom (UK) investigated the views on passive smoking in children aged 4-8 years using "Draw and Write" exercises and semi-structured interviews ${ }^{7}$. The children expressed concerns about both the smokers' and their own health and had strong negative feelings about being exposed to tobacco smoke. Another UK study investigated the accounts of family members' smoking by adolescents aged 10-15 years using individual and group interviews ${ }^{8}$. This study found that adolescents strongly disliked family members' tobacco use, and they expressed health concerns more about their family members that use tobacco than themselves.

Another study using ethnographic interviews in adolescents aged 11-19 years in Canada also found a strong dislike of parental smoking, and serious concerns about the health of 
their parents and themselves ${ }^{9}$. The adolescents recounted their unpleasant experiences of being exposed to SHS and how parent-child relationships were affected. To conclude, these studies generally suggested that children and adolescents' perceptions of parental smoking were negative.

These qualitative studies have provided valuable in-depth descriptions of children and adolescents' perceptions, but also had a few limitations. First, their generalisability may be limited, given their non-probabilistic and relatively small samples. Moreover, whether the negative perceptions found in the West can be safely generalised to China is uncertain. Unlike Western families, Chinese families tend to be hierarchical based on generation and age, due to the influence of Confucianism ${ }^{10}$. Chinese society also has a norm against female smoking11. In Hong Kong, the daily smoking prevalence in 2012 was $19.1 \%$ in males, but only $3.1 \%$ in females ${ }^{12}$. These social and cultural factors may influence the perceptions of paternal and maternal smoking in Chinese children and adolescents. In addition, although one of the studies found that younger age was associated with negative perceptions $^{7}$, more detailed investigations of the associated factors should rely on quantitative data.

Understanding children's perceptions of parental smoking may have implications for the design of family-based smoking cessation and SHS reduction programmes, and is also relevant to a recently discovered issue that tobacco use may adversely affect the interpersonal well-being of a family ${ }^{13}$. Using the data of a large-scale cross-sectional survey with a representative sample of secondary students in Hong Kong, the most urbanized city of China, we investigated the negative perceptions of paternal and maternal smoking in adolescents, and the factors associated with the negative perceptions.

\section{METHODS}

\section{Survey design and ethics statement}

A cross-sectional survey of secondary school students in Hong Kong was conducted in 2010-11. A random sample of secondary schools stratified by districts was used, with all the students in chosen schools invited. The survey was conducted in classrooms using a questionnaire and an anonymous answer sheet. Teachers maintained classroom order and were instructed to avoid patrolling near students. Returned answer sheets were sealed in an opaque envelope in front of students on completion.

A total of 61,810 students from 79 secondary schools returned valid answer sheets, with student and school level response rates of $97.3 \%$ and $25.8 \%$. The surveyed schools were similar with those not surveyed (ie, those declined participation or not invited) with regard to district (Chi-square, $\mathrm{P}=0.91$ ), sex composition (Chi-square, $\mathrm{P}=0.53$ ) and medium of instruction (Chi-square, $\mathrm{P}=0.27$ ). More details of the survey methods have been reported elsewhere ${ }^{14}$.

Invitation letters were sent to parents via students. No reply was requested, and the parents who declined participation could ask their children to return a blank answer sheet. Even with parental consent, student participation was voluntary. Ethics approval was granted by the Institutional Review Board of the University of Hong Kong/Hospital Authority Hong Kong West Cluster.

\section{Measurement}

A Chinese version of the questionnaire was used in most of the schools, and an English version was used in a few English-medium schools (2.5\%). The questionnaire items (in Chinese and English) used in the present study are shown in Supplementary Online Material. Negative perceptions of parental smoking for each parent were assessed using two questions: (1) "do you think it is acceptable if your father/mother smokes?" (considering paternal/maternal smoking unacceptable) with options dichotomised as "yes" (unacceptable/very unacceptable) and "no" (very acceptable/ acceptable); (2) "would you mind letting your classmates or friends know if your father/mother smokes?" (minding peers knowing paternal/maternal smoking) with options also dichotomised as "yes" (really mind/mind) and "no" (don't mind/really don't mind). Two binary indicators of the negative perceptions were thus derived for each parent. Paternal (no/ yes) and maternal (no/yes) smoking were also reported.

Age (in years), sex and perceived family affluence (relatively poor/poor to average/average/average to rich/relatively rich) were reported, with age dichotomised as " $\varsigma 14$ " and " 15 years old" and perceived family affluence as "relatively poor or poor to average" and "average or above".

Two questions assessed the awareness of the harm of tobacco: (1) "do you think smoking cigarettes is harmful to your health?"; (2) "do you think breathing secondhand smoke will harm your health?" Each had response options of "definitely not", "probably not", "probably yes" and "definitely yes", and those choosing "definitely yes" were deemed certain about the respective harm.

One question assessed peer smoking: "do any of your good friends smoke cigarettes?" with response options of "none", "some", "half", "most" and "all". Options other than "none" were deemed affirmative. Students were also asked: "in how many of the past 30 days did you smoke cigarettes?" with response options of "0", “1-2", “3-5”, “6-9”, "10-19”, "20-29” 
and "30 days". Those choosing any options other than " 0 " were classified as smoking in the past 30 days.

Students also reported whether their parents smoked near them at home (no/yes) and outside home (no/yes) in the past 7 days, with separate items for the father and mother, and the number of co-residing smokers $(0 / 1 / 2 / 3 / 4 / 5$ or more). The students with paternal/maternal smoking and 2 or more co-residing smokers were classified as having ( 1 or more) co-residing smokers in addition to the father/mother. The students who reported that their father/mother smoked near them either at home or outside home in the past 7 days were classified as being exposed to SHS from the father/mother.

\section{Statistical analysis}

The proportion of students considering paternal smoking unacceptable and the proportion minding peers knowing paternal smoking were calculated in students with paternal smoking; the corresponding proportions for maternal smoking were calculated in students with maternal smoking. These proportions and all other descriptive results were weighted by age, sex and grade distributions of the corresponding population using data provided by the Education Bureau of the Hong Kong Government. The factors associated with the indicators of adolescents' negative perceptions of paternal and maternal smoking were respectively explored in students with paternal and maternal smoking. Random-effects multivariable Poisson regression models yielded adjusted prevalence ratios (PRs) of each indicator for sex, age, perceived family affluence, being certain about the harm of smoking and SHS, peer smoking, smoking in the past 30 days, having co-residing smokers in addition to the father/mother and SHS from the father/mother in the past 7 days, with these factors adjusted for each other. PRs were used instead of odds ratios (ORs) because preliminary analyses indicated that the outcomes were prevalent, and the ORs in this case, if interpreted as approximations of PRs, would be misleading. Poisson regression with robust variance estimators was used to estimate PRs and their 95\% confidence intervals (CIs) for the cross-sectional data by treating the binary outcomes as count variables and assuming all of the subjects have the same length of follow-up ${ }^{15}$. The random effects were used to adjust for potential school clustering effects. All analyses were conducted using STATA 13.0.

\section{RESULTS}

Table 1 indicates that, overall, students' mean age (standard deviation) was 14.6 (2.0) years, and 50.8\% were boys. Paternal smoking $(29.8 \%)$ was much more common than maternal smoking (5.7\%). Of those who reported paternal smoking, $50.8 \%$ considered it unacceptable, and $21.0 \%$ minded if their peers knew of it. Of those who reported maternal smoking, $48.1 \%$ considered it to be unacceptable, and $30.4 \%$ minded if their peers knew of it.

Table 2 indicates that gender was not associated with the two indicators of negative perceptions of maternal smoking. Although gender was weakly associated with the two indicators for paternal smoking, the direction of the two associations was not consistent. Generally, older age was negatively associated with the indicators of negative perceptions of parental smoking. For example, being 15 years old or above was associated with an adjusted prevalence ratio (aPR) of 0.89 (95\% confidence interval 0.87-0.92) for considering paternal smoking unacceptable, compared with those younger. Higher perceived family affluence was negatively associated with considering paternal smoking unacceptable (aPR 0.88, 0.86-0.91) and considering maternal smoking unacceptable (aPR 0.86, 0.81-0.92); although it was positively associated with minding peers knowing of their paternal smoking, the corresponding association for maternal smoking was nonsignificant.

Generally, being certain about the harm of smoking and SHS were positively associated with the indicators of negative perceptions of parental smoking (Table 2). For example, being certain about the harm of smoking was associated with an aPR of 1.33 (1.25-1.41) for considering paternal smoking unacceptable. Peer smoking was negatively associated with the indicators of negative perceptions of parental smoking. For example, it was associated with an aPR of 0.82 (0.79-0.85) for considering paternal smoking unacceptable. Smoking in the past 30 days was negatively associated with considering paternal smoking unacceptable, but the corresponding associations for the other indicators were non-significant.

Table 2 also indicates that, compared with having the father as the only co-residing smoker, having additional co-residing smokers was negatively associated with considering paternal smoking unacceptable (aPR 0.84, 0.81-0.88). Similarly, having co-residing smokers in addition to the mother was negatively associated with considering maternal smoking unacceptable (aPR 0.87, 0.81-0.94). The corresponding associations for minding peer knowing of parental smoking were non-significant. Generally, SHS exposure from a parent was negatively associated with the indicators of negative perceptions of the parent's smoking. For example, SHS exposure from the father was associated with an aPR of 0.90 (0.85-0.95) for minding peers knowing of paternal smoking. An exception was the weak positive association between SHS 
Table 1. Characteristics of the survey sample, students with paternal smoking, and students with maternal smoking, Hong Kong 2010-2011

\begin{tabular}{|c|c|c|c|}
\hline & Entire sample $(n-61,810)$ & $\begin{array}{l}\text { Students with paternal smoking } \\
\left(\mathrm{n}=19,181,29.8^{\circ}\right)\end{array}$ & $\begin{array}{l}\text { Students with maternal } \\
\text { smoking }\left(n-3,678,5.7^{\circ} \%\right)\end{array}$ \\
\hline Variable & $\mathrm{N}(\%) a$ & $\mathrm{~N}(\%) a$ & $\mathrm{~N}(\%) a$ \\
\hline Mean age in years (standard deviation) & $14.6(2.0)$ & $14.7(2.0)$ & $14.4(1.9)$ \\
\hline Age, years $(\geq 15)$ & $33715(51.0)$ & $10527(51.3)$ & $1739(45.0)$ \\
\hline $\begin{array}{l}\text { Perceived family affluence (Average or } \\
\text { above) }\end{array}$ & $41481(68.7)$ & $12034(64.3)$ & $2379(65.7)$ \\
\hline Being certain about the harm of smoking & $54038(87.8)$ & $16483(86.1)$ & $2857(78.2)$ \\
\hline Being certain about the harm of SHS & $47889(78.0)$ & $14463(75.5)$ & $2449(67.1)$ \\
\hline Peer smoking & $26457(41.5)$ & $9645(49.3)$ & $2321(61.7)$ \\
\hline Smoking in the past 30 days & $3921(5.9)$ & $1488(7.3)$ & $719(18.7)$ \\
\hline $\begin{array}{l}\text { Co-residing smokers in addition to the } \\
\text { father }\end{array}$ & - & $3623(20.4)$ & - \\
\hline SHS from the father in the past 7 days & - & $11883(61.7)$ & - \\
\hline SHS from the mother in the past 7 days & - & - & $2155(58.2)$ \\
\hline Considering paternal smoking unacceptable & - & $9830(50.8)$ & - \\
\hline Considering maternal smoking unacceptable & - & - & $1807(48.1)$ \\
\hline Minding peers knowing of paternal smoking & - & $3904(21.0)$ & - \\
\hline Minding peers knowing of maternal smoking & & & $1112(30.4)$ \\
\hline
\end{tabular}

Table 2. Factors associated with negative perceptions of paternal and maternal smoking among secondary school students, Hong Kong 2010-11

Studentswithpaternalsmoking (n=19,181) Considering paternal V Vinding peersknowing smoking unacceptable

Factors $^{a}$ Adjusted PRsb (95\% Cl) of their paternal smoking

Sex (Male)

$0.97(0.95-1.00)$

Age, years (>15)

Perceived family affluence (Average or above)

Certain about the harm of $1.33(1.25-1.41)$ smoking (Yes)

Certain about the harm of SHS (Yes)

Peer smoking (Yes)

Smoking in the past 30 days (Yes)

Co-residing smokers in addition to the father (Yes)

Co-residing smokers in addition to the mother (Yes)

SHS from the father in the $1.05(1.02-1.08)$ past 7 days (Yes)

SHS from the mother in the past 7 days (Yes) ( $95 \% \mathrm{CI}$ )

$1.11(1.03-1.20)$

$0.89(0.87-0.92)$

$0.62(0.58-0.66)$

$1.10(1.03-1.17)$

$1.03(0.92-1.14)$

$1.60(1.52-1.69)$

$1.31(1.20-1.43)$

$0.82(0.79-0.85)$

$0.56(0.52-0.60)$

$0.53(0.46-0.60)$

$1.03(0.87-1.22)$

$0.84(0.81-0.88)$

$1.00(0.93-1.09)$

$0.90(0.85-0.95)$
Students with maternalsmoking $(n=3,678)$

Consideringmaternal Vinding peersknowing of smoking unacceptable theirmaternalsmoking

Adjusted PRsb Adjusted PRsb Adjusted PRsb
$(95 \% \mathrm{CI})$ $(95 \% \mathrm{CI})$

$1.03(0.97-1.09)$

$1.01(0.90-1.13)$

$0.94(0.87-1.01)$

$0.80(0.70-0.90)$

$0.86(0.81-0.92)$

$1.01(0.91-1.12)$

$1.43(1.24-1.66)$

$1.13(0.96-1.34)$

$1.28(1.15-1.42)$

$1.23(1.05-1.43)$

$0.83(0.78-0.89)$

$0.63(0.56-0.72)$

$0.87(0.75-1.00)$

$1.15(0.97-1.35)$

$0.87(0.81-0.94)$

$0.97(0.87-1.08)$

$0.90(0.85-0.96)$

$0.91(0.84-0.99)$

${ }^{\circ}$ The factor in brackets is the index level (eg. Male gender) ${ }^{b}$ Poisson regression model with robust variance estimator was used, with adjustment of school clustering effect and mutual adjustment of sex, age, perceived family affluence, being certain about the harm of smoking, being certain about the SHS, peer smoking, smoking in the past 30 days, having co-residing smokers in addition to the father/mother and SHS from the father/mother in the past 7 days. 
exposure from the father and considering paternal smoking unacceptable (aPR 1.05, 1.02-1.08).

\section{DISCUSSION}

We found that about half the Hong Kong adolescents with a smoking parent considered the parent's smoking unacceptable; while about a quarter minded if their peers knew of the parent's smoking status, the proportion was higher for maternal smoking than paternal smoking. These results reflect an influence of the social norm against female smoking but also indicate that, despite such a norm, adolescents consider paternal and maternal smoking equally unacceptable. Generally, our results are compatible with qualitative studies in the West which showed that children and adolescents strongly disliked family members' smoking ${ }^{7-9}$. Our results indicate that the negative perceptions of parental smoking were common in Hong Kong adolescents.

Notably, two of the aforementioned qualitative studies reported frictions and unpleasantness between children and parents triggered by parental smoking, ${ }^{8,9}$, and one further reported adverse effects on parent-child relationships ${ }^{9}$. This was echoed in a population study in Hong Kong children, which found that half the children in smoking families reported unpleasant experiences caused by smoking or SHS at home in the past 30 days, and that family members' smoking and exposure to SHS at home were both associated with family unhappiness ${ }^{13}$. These findings together with the prevalent negative perceptions of parental smoking found in the present study provide preliminary but important evidence that tobacco use may affect the interpersonal well-being of a family. Although such impact needs further investigation, the finding of the present study may be communicated to smoking parents as a novel tobacco control message.

A qualitative study documented the actions children took towards their parents' smoking, including mediating smoking risk messages, expressing concern, and hiding or destroying their parents' cigarettes ${ }^{8}$. Such finding together with the prevalent negative perceptions of parental smoking found in the present study suggests that adolescents may be willing to be involved in family-based smoking cessation programmes for parents. The feasibility and effectiveness of such programmes should be explored in future research.

The prevalent negative perceptions of parental smoking also suggest that future health education programmes may need to help adolescents deal with family members' smoking positively and to emphasise a notion that successful cessation can be facilitated by a loving and supportive family ${ }^{16}$. Moreover, given the significant proportion of adolescents who minded if their peers knew of their parents' smoking, it is also important that future tobacco-related health education programmes for adolescents should be developed in a non-stigmatising way.

In the present study, older adolescents were generally less likely to have negative perceptions of parental smoking. This association was also reported in a study of UK children aged 4-87. These findings suggest that, as children and adolescents grow older, they may get more used to parental smoking or become more receptive to smoking in general. Wealthier adolescents were less likely to consider parental smoking unacceptable. It is well known that, in poor families, tobacco expenditure crowds out the expenditure on other household essentials $^{17-19}$. This may exacerbate poor adolescents' negative perceptions of parental smoking.

Generally, adolescents who were certain about the harm of tobacco were more likely to have negative perceptions of parental smoking, suggesting that health concerns may contribute to the negative perceptions. Adolescents with peer smoking were less likely to have negative perceptions of parental smoking, suggesting that the presence of smoking close friends may influence adolescents' perceived smoking norms and thus moderate the negative perceptions. However, it was also possible that a general anti-smoking attitude led to both the negative perceptions and a tendency to avoid socialising with smokers.

Adolescents with co-residing smokers in addition to a parent were less likely to consider the parent's smoking unacceptable. This suggests that the presence of more co-residing smokers may influence adolescents' perceived smoking norms and thus moderate their negative perceptions of parental smoking. Generally, students exposed to SHS from a parent were less likely than those unexposed to have negative perceptions of the parent's smoking. It was possible that adolescents with the negative perceptions were more likely to avoid SHS from their parents or to require their parents not to smoke near them.

The associations of adolescents' negative perceptions with being certain about the harm of tobacco, fewer co-residing smokers and no smoking peers suggest that, if the awareness of the harm of tobacco continues to increase, and smoking prevalence to decrease, the negative perceptions may become more prevalent over time.

A strength of the present study is its large sample size, which allows the investigation of the factors associated with the negative perceptions of parental smoking, even the prevalence of maternal smoking is very low. However, there are also several limitations. First, it was relatively common for schools to decline participation, which resulted in a low school level response rate $(25.8 \%)$. However, the main reason of refusal 
was the difficulty in finding school time for the survey, and the surveyed schools were similar with those not surveyed with regard to key indicators of socio-economic status (ie, district $\&$ medium of instruction). Therefore, the results yielded by our sample should be reasonably representative of the population of Hong Kong secondary school students. Another limitation is the external validity. Hong Kong has one of the lowest adult daily smoking prevalence (10.7\% in 2012) in the developed world and many stringent tobacco control policies, which include smoke-free legislation for indoor public places, high tobacco duty, prohibition of tobacco advertising, etc. ${ }^{20-}$ 22. Therefore, the findings of the present study may not be generalised to vastly different settings, especially those with inadequate tobacco control measures.

\section{CONCLUSIONS}

About half the Hong Kong adolescents with a smoking parent considered the parent's smoking unacceptable, and about a quarter minded if their peers knew of their parent's smoking. Such negative perceptions were more common in adolescents who were certain about the harm of tobacco, and had fewer co-residing smokers and no smoking peers.

\section{REFERENCES}

1. U.S. Department of Health and Human Services. The health consequences of involuntary exposure to tobacco smoke: a report of the Surgeon General. Available from: http://www.ncbi.nlm.nih.gov/ books/NBK44324/pdf/Bookshelf_NBK44324.pdf. (accessed April 2016)

2. Chen JJ, Ho SY, Wang MP, Lam TH. Reactions to thirdhand smoke are associated with openness to smoking in young never smoking children. J Community Health 2015;41:461-67 doi: 10.1007/s10900-015-0115-0.

3. Lessov-Schlaggar CN, Wahlgren DR, Liles S, et al. Sensitivity to secondhand smoke exposure predicts future smoking susceptibility. Pediatrics 2011;128:254-62 doi: 10.1542/peds.2010-3156.

4. Wang MP, Ho SY, Lam TH. Parental smoking, exposure to secondhand smoke at home, and smoking initiation among young children. Nicotine Tob Res 2011;13:827-32 doi: $10.1093 / \mathrm{ntr} / \mathrm{ntr} 083$.

5. Mak KK, Ho SY, Day JR. Smoking of parents and best friendindependent and combined effects on adolescent smoking and intention to initiate and quit smoking. Nicotine Tob Res 2012;14:1057-64 doi: 10.1093/ntr/nts008.

6. Doll R, Peto R, Boreham J, Sutherland I. Mortality in relation to smoking: 50 years' observations on male British doctors. BMJ 2004;328:1519 doi: 10.1136/bmj.38142.554479.AE

7. Woods SE, Springett J, Porcellato L, Dugdill L. 'Stop it, it's bad for you and me': experiences of and views on passive smoking among primary-school children in Liverpool. Health Educ Res 2005;20:645-55 doi: 10.1093/her/cyh027.

8. Rowa Dewar N, Amos A, Cunningham Burley S. Children's resistance to parents' smoking in the home and car: a qualitative study. Addiction 2014;109:645-52 doi: 10.1111/add.12435.

9. Woodgate RL, Kreklewetz CM. Youth's narratives about family members smoking: parenting the parent-it's not fair! BMC Public Health 2012;12:965 doi: 10.1186/1471-2458-12-965.

10. Yang R, Neal AG. The impact of globalization on family relations in China. Int J Sociol Fam 2006;32:113-26.

11. Sansone N, Yong H-H, Li L, Jiang Y, Fong GT. Perceived acceptability of female smoking in China: findings from waves 1 to 3 of the ITC China Survey. Tob Control 2015;24:iv48-54 doi:10.1136/tobaccocontrol-2015-052380.

12. Info-Station. Tobacco Control Office, Department of Health, The Government of the Hong Kong Special Administrative Region. Available from: https://www.tco.gov.hk/english/infostation/ infostation_sta_01.html. Updated December 13, 2013. (accessed April 2016)

13. Chen JJ, Ho SY, Au WM, Wang MP, Lam TH. Family Smoking, Exposure to Secondhand Smoke at Home and Family Unhappiness in Children. Int J Environ Res Public Health 2015;12:14557-70 doi: 10.3390/ijerph121114557.

14. Leung LT, Ho SY, Wang MP, Lo WS, Lam TH. Exposure to secondhand smoke from neighbours and respiratory symptoms in never-smoking adolescents in Hong Kong: a cross-sectional study. BMJ Open 2015;5:e008607 doi: 10.1136/bmjopen-2015-008607.

15. Barros AJ, Hirakata VN. Alternatives for logistic regression in crosssectional studies: an empirical comparison of models that directly estimate the prevalence ratio. BMC Med Res Methodol 2003;3:21 doi: 10.1186/1471-2288-3-21.

16. Roski J, Schmid LA, Lando HA. Long-term associations of helpful and harmful spousal behaviors with smoking cessation. Addict Behav 1996;21:173-85 doi:10.1016/0306-4603(95)00047-X.

17. John RM, Ross H, Blecher E. Tobacco expenditure and its implications for household resource allocation in Cambodia. Tob Control 2012;21:341-6 doi: 10.1136/tc.2010.042598.

18. John RM. Crowding out effect of tobacco expenditure and its implications on household resource allocation in India. Soc Sci Med 2008;66:1356-67 doi: 10.1016/j.socscimed.2007.11.020.

19. Xin Y, Qian J, Xu L, et al. The impact of smoking and quitting on household expenditure patterns and medical care costs in China. Tob Control 2009;18:150-5 doi: 10.1136/tc.2008.026955.

20. Ho SY, Wang MP, Lo WS, et al. Comprehensive smoke-free legislation and displacement of smoking into the homes of young children in Hong Kong. Tob Control 2010;19:129-33 doi: 10.1136/tc.2009.032003.

21. Legislative Council of Hong Kong. Smoking (Public Health) Ordinance. Available from: http:// www.legislation.gov.hk/blis_pdf. nsf/6799165D2FEE3FA9482575 5E0033E532/FC20BFF93D75AD CC482575EE00764F46/\$FILE/ CAP_371_e_b5.pdf. Published September 2, 2012. (accessed April 2016)

22. Legislative Council of Hong Kong. Bills committee on dutiable commodities (Amendment) Bill 2014. Available from: http://www. legco.gov.hk/yr13-14/english/bc/ bc05/papers/bc050408cb1-11982-e.pdf. Published 2014. (accessed April 2016)
ACKNOWLEDGEMENTS

We thank the schools and students for participating in the survey.

CONFLICT OF INTEREST All the authors have completed and submitted the ICME Form for Disclosure of Potential Conflicts of Interest and none were reported.

\section{FUNDING}

This work was supported by the Food and Health Bureau of the Government of the Hong Kong Special Administrative Region.

PROVENANCE AND PEER REVIEW

Not commissioned; Externally peer reviewed 